\title{
Erratum to: Multiple sign-changing solutions for a semilinear Neumann problem and the topology of the configuration space of the domain boundary
}

\author{
Naoki Shioji
}

Published online: 20 October 2010

C) Springer-Verlag 2010

Erratum to: Calc. Var. (2009)

DOI 10.1007/s00526-009-0288-4

We would like to modify the following three parts.

First, since it seems difficult to show $\varepsilon_{0} / 2 \leq\left\|\varphi\left(t_{2}, u\right)-\varphi\left(t_{1}, u\right)\right\|$ in the proof of Lemma 32, we modify as follows:

(E.1) Change the definition of $\mathcal{M}_{d}$ in p. 342 to $\mathcal{M}_{d}=\left\{u \in H_{d}: \Phi_{d}(u) \leq 3 c_{0}, u^{+}, u^{-} \in\right.$ $\left.\mathcal{N}_{d}\right\}$.

(E.2) Remove the assumption

$$
\left|\left(\nabla \Phi_{d_{n}}\left(u_{n}\right), u_{n}\right)_{H_{d_{n}}}\right| \leq 1 \text { for all } n \in \mathbb{N}
$$

from Proposition 5.

(E.3) Change the definition of $\mathcal{U}_{d, \varepsilon}$ in p. 344 to $\mathcal{U}_{d, \varepsilon}=\left\{u \in H_{d}: \operatorname{dist}_{H_{d}}\left(u, \mathcal{M}_{d}\right) \leq \varepsilon\right\}$,

(E.4) Remove Lemma 22.

Consequently, we have the following.

(i) Even without the assumption (0.1) in the proof of Proposition 5, we can show the boundedness of $\left\{\left\|u_{n}\right\|_{H_{d_{n}}}\right\}$. Indeed, since we can chose $\left\{\widetilde{u}_{n}\right\}$ such that $\widetilde{u}_{n} \in \mathcal{M}_{d_{n}}$ and $\left\|u_{n}-\widetilde{u}_{n}\right\|_{H_{d_{n}}} \rightarrow 0$, we have $\Phi_{d_{n}}\left(\widetilde{u}_{n}\right) \leq 3 c_{0}$ for each $n \in \mathbb{N}$, and hence $\left\{\left\|\widetilde{u}_{n}\right\|_{H_{d_{n}}}\right\}$ is bounded by Lemma 17. From $\left\|u_{n}-\widetilde{u}_{n}\right\|_{H_{d_{n}}} \rightarrow 0,\left\{\left\|u_{n}\right\|_{H_{d_{n}}}\right\}$ is also bounded.

(ii) As a result of (i), the proofs in Lemmas 23 and 24 work without the assumption (0.1).

Communicated by H. Brezis.

The online version of the original article can be found under doi:10.1007/s00526-009-0288-4 .

N. Shioji $(\varangle)$

Department of Mathematics, Graduate School of Environment and Information Sciences,

Yokohama National University, Tokiwadai, Hodogaya-ku, Yokohama 240-8501, Japan

e-mail: shioji@math.sci.ynu.ac.jp 
(iii) In the proof of Lemma 26, we do not need the assumption (0.1) or Lemma 22 either. Indeed, since we have changed the definition of $\mathcal{M}_{d}$, we can show the boundedness of $\left\{\left\|u_{n}\right\|_{H_{d_{n}}}\right\}$ by a similar way as in (i).

(iv) In order to show $\mathcal{A}$ is bounded, Lemma 22 was used in the last line of p. 349. However, we can show its boundedness by Lemma 15 .

(v) Then the proof of Lemma 32 works, i.e., we can show $\varepsilon_{0} / 2 \leq\left\|\varphi\left(t_{2}, u\right)-\varphi\left(t_{1}, u\right)\right\|$.

Next, it seems difficult to show the existence of $\left\{v_{n}\right\} \subset \operatorname{Inv}\left(\Phi_{d}^{\mu_{0}} \backslash \mathcal{E}_{T_{0}}\right) \backslash U_{1}$ satisfying $\Phi_{d}\left(v_{n}\right) \rightarrow \mathfrak{c}$ and $\left(1+\left\|v_{n}\right\|_{H_{d}}\right)\left\|\nabla \Phi_{d}\left(v_{n}\right)\right\|_{H_{d}} \rightarrow 0$ in the proof of Proposition 6. Indeed, from $\frac{2}{n} \geq \frac{1}{16} \int_{0}^{t_{n}} \theta\left(\varphi\left(t, u_{n}\right)\right) \min \left\{1,\left(1+\left\|\varphi\left(t, u_{n}\right)\right\|_{H_{d}}\right)^{2}\left\|\nabla \Phi_{d}\left(\varphi\left(t, u_{n}\right)\right)\right\|_{H_{d}}^{2}\right\} d t$ in p. 351, we might only extract $\left\{s_{n}\right\}$ such that $s_{n} \in\left[0, t_{n}\right]$ and $\theta\left(\varphi\left(s_{n}, u_{n}\right)\right) \rightarrow 0$, which only might show the existence of $\left\{w_{n}\right\} \subset H_{d} \backslash \widetilde{H}_{d}$ with $\left\|\varphi\left(s_{n}, u_{n}\right)-w_{n}\right\|_{H_{d}} \rightarrow 0$. However, it might hold that $\Phi_{d}\left(w_{n}\right) \rightarrow \infty$. In this case, it seems difficult to obtain a contradiction. So we modify as follows:

(E.5) We change the definition of $\theta_{1}$ in p. 348 to $\theta_{1}(u)=\min \left\{\operatorname{dist}_{H_{d}}\left(u, \Phi_{d}^{3 c_{d}} \backslash \widetilde{H}_{d}\right), 1\right\}$ and we define $\theta$ and $W$ as in the paper.

(E.6) Instead of Lemma 29, we consider that $\varphi$ is a semiflow such that $\varphi:[0, \infty) \times$ $\left\{u \in H_{d}: \Phi_{d}(u)<3 c_{d}\right\} \rightarrow\left\{u \in H_{d}: \Phi_{d}(u)<3 c_{d}\right\}$ defined by $\varphi(0, u)=u$, $\partial \varphi / \partial t(t, u)=-W(\varphi(t, u))$ for $(t, u) \in[0, \infty) \times H_{d}$ with $\Phi_{d}(u)<3 c_{d}$. The proof of Lemma 29 works for the existence such $\varphi$.

(E.7) We change the definition of $\mathcal{E}_{T}$ in p. 349 to $\mathcal{E}_{T}=\left\{u \in H_{d}: \Phi_{d}(u)<3 c_{d}\right.$ and there exists $t \in[0, T]$ such that $\left.\Phi_{d}(t, u) \in \mathcal{D}_{d, a_{0}} \cup \Phi_{d}^{c_{d}-\delta_{0}}\right\}$.

(E.8) We change Lemma 31 as follows: For each $T>0, \Phi_{d}^{\mu_{0}} \cup \mathcal{E}_{T}$ is a strictly positively invariant, closed subset of $\left\{u \in H_{d}: \Phi_{d}(u)<3 c_{d}\right\}$ and $\mathcal{E}_{T}$ is a strictly positively invariant, closed subset of $\Phi_{d}^{\mu_{0}} \cup \mathcal{E}_{T}$.

Finally, in the statement of Theorem, it was said that if $\Omega$ is bounded then there is at least one other sign-changing solution which has at most four nodal domains. The proof in the last section works for the existence of at least one other sign-changing solution, but it seems difficult to show that its nodal domains are at most four. Because it seems difficult to show $l\left([0,2] \times C_{\rho}(\partial \Omega) \times I_{b}^{2}\right) \subset \Phi_{d}^{\mu_{1}}$, which was written in p. 355, 1.17个. In the case $2 \max \left\{c_{0,+}, c_{0,-}\right\}<3 \min \left\{c_{0,+}, c_{0,-}\right\}$, letting $x_{*}$ be a point in $\Omega$ such that $\operatorname{dist}_{\mathbb{R}^{N}}\left(x_{*}, \partial \Omega\right) \geq$ $5 \rho \bar{\rho}$ and $\left|x_{*}-x_{\sharp}\right| \geq 5 \rho \bar{\rho}$ and by the same proof, we can show that the solution has at most four nodal domains. In the case $2 \max \left\{c_{0,+}, c_{0,-}\right\} \geq 3 \min \left\{c_{0,+}, c_{0,-}\right\}$, letting $k \in \mathbb{N}$ be the least integer such that $2 \max \left\{c_{0,+}, c_{0,-}\right\}<k \min \left\{c_{0,+}, c_{0,-}\right\}$, we can only show that the other solution has at most $k+1$ nodal domains. 